\title{
Hamiltonian engineering via invariants and dynamical algebra
}

\author{
E. Torrontegui, ${ }^{1,2}$ S. Martínez-Garaot, ${ }^{2}$ and J. G. Muga ${ }^{2,3}$ \\ ${ }^{1}$ Institute of Chemistry, The Hebrew University, Jerusalem 91904, Israel \\ ${ }^{2}$ Departamento de Química Física, Universidad del País Vasco UPV/EHU, Apartado 644, Bilbao, Spain \\ ${ }^{3}$ Department of Physics, Shanghai University, 200444 Shanghai, People's Republic of China
}

(Received 23 February 2014; published 14 April 2014)

\begin{abstract}
We use the dynamical algebra of a quantum system and its dynamical invariants to inverse engineer feasible Hamiltonians for implementing shortcuts to adiabaticity. These are speeded up processes that end up with the same populations as slow, adiabatic ones. As application examples, we design families of shortcut Hamiltonians that drive two- and three-level systems between initial and fina configurations imposing physically motivated constraints on the terms (generators) allowed in the Hamiltonian.
\end{abstract}

DOI: 10.1103/PhysRevA.89.043408

PACS number(s): $32.80 . \mathrm{Qk}, 42.50 . \mathrm{Dv}, 03.65 .-\mathrm{w}, 03.67 .-\mathrm{a}$

\section{INTRODUCTION}

The current development of "shortcuts to adiabaticity" to speed up adiabatic, slow processes in different field (trap expansions [1-4], atom or ion transport [5-10], internal state control [11-18], wave-packet splitting [19-25], many-body state engineering [26-31], optics [32], cooling methods [3335], and cooling cycles and quantum engines [36-39]) raises a number of practical and fundamental questions (see [40] for a recent review). An important one is how to generate alternative shortcuts when the, generally time-dependent, Hamiltonian that speeds up the slow process is difficul or impossible to realize in the laboratory [30,31,40-43]. Typically, the difficultie are related to specifi terms that cannot be implemented. Several examples have shown that the symmetry of the Hamiltonian is instrumental in designing feasible alternative Hamiltonians (and shortcuts) that keep the same population dynamics in some basis or at least the same fina populations [40-42]. However, a systematic symmetry-based approach to inverse engineering the Hamiltonian, given the desired dynamics and specifi constraints imposed on its structure, has been lacking. In this paper, we provide basic elements for such an approach and set the inverse problem from a (Lewis and Riesenfeld [44]) dynamical invariant to the Hamiltonian, assuming that both are members of a dynamical Lie algebra, i.e., a vector space spanned by operators (generators) closed under commutation. Dynamical invariants correspond to operators whose expectation values remain constant for states evolving with the associated Hamiltonian (they may or may not commute with it). Invariants that belong to the dynamical Lie algebra of the Hamiltonian have been used to solve the dynamics or to calculate geometric phases [45-48]. Since the dynamical invariants contain information about the system evolution (any density operator describing its evolution is a dynamical invariant), they have also been used to design shortcuts to adiabaticity $[1-4,7-10,18,25-29,39,40,49]$, as we shall do here, taking explicitly into account the dynamical algebra in the Hamiltonian construction.

In Sec. II, a construction method is provided to design families of Hamiltonians for a given invariant in the space spanned by their corresponding algebra. This method allows one to impose constraints on the generators, such as making some of them zero. In addition, boundary conditions for the invariant are specifie at initial and fina instants so that the Hamiltonian indeed drives the system along a shortcut to adiabaticity without fina excitation. We work out two examples that illustrate the construction algorithm. In Sec. III, we construct real Hamiltonians within the SU(2) algebra to drive a two-level atom state without using the Pauli matrix $\sigma_{y}$. Then we analyze, in Sec. IV, a three-level system described by a four-dimensional Lie algebra, with the goal of achieving fast "insulator-superfluid transitions for two interacting bosons in two wells, using only two feasible generators. Conclusions and open questions are summarized in Sec. V. The Appendix provides an alternative approach, using Gaussian elimination, to the operational approach of Sec. II.

\section{GENERAL FORMALISM}

Let us assume that the time-dependent Hamiltonian $H(t)$ describing a quantum system is given by a linear combination of Hermitian "generators" $T_{a}$,

$$
H(t)=\sum_{a=1}^{N} h_{a}(t) T_{a},
$$

where the $h_{a}(t)$ are real time-dependent functions and the $T_{a}$ span a Lie algebra (dynamical algebra [45]),

$$
\left[T_{b}, T_{c}\right]=\sum_{a=1}^{N} \alpha_{a b c} T_{a},
$$

where the $\alpha_{a b c}$ are the "structure constants." Associated with the Hamiltonian, there are time-dependent Hermitian invariants of motion $I(t)$ that satisfy [44]

$$
\frac{d I}{d t} \equiv \frac{\partial I(t)}{\partial t}-\frac{1}{i \hbar}[H(t), I(t)]=0 .
$$

A wave function $|\Psi(t)\rangle$ which evolves with $H(t)$ can be expressed as a linear combination of invariant modes [44],

$$
|\Psi(t)\rangle=\sum_{n} c_{n} e^{i \alpha_{n}}\left|\phi_{n}(t)\right\rangle,
$$

where the $c_{n}$ are constants, the phases $\alpha_{n}$ fulfil

$$
\hbar \frac{d \alpha_{n}}{d t}=\left\langle\phi_{n}(t)\left|i \hbar \frac{\partial}{\partial t}-H(t)\right| \phi_{n}(t)\right\rangle,
$$


and the eigenvectors of $I(t),\left|\phi_{n}(t)\right\rangle$, are assumed to form a complete set and satisfy

$$
I(t)\left|\phi_{n}(t)\right\rangle=\lambda_{n}\left|\phi_{n}(t)\right\rangle,
$$

with $\lambda_{n}$ being the constant eigenvalues.

If the invariant is a member of the dynamical algebra, it can be written as

$$
I(t)=\sum_{a=1}^{N} f_{a}(t) T_{a},
$$

where $f_{a}(t)$ are real, time-dependent functions. Note that some $f_{a}$ or some $h_{a}$ may be zero. Replacing Eqs. (1) and (7) into Eq. (3), and using Eq. (2), the functions $h_{a}(t)$ and $f_{a}(t)$ satisfy $[45,50]$

$$
\dot{f}_{a}(t)-\frac{1}{i \hbar} \sum_{b=1}^{N} \sum_{c=1}^{N} \alpha_{a b c} h_{b}(t) f_{c}(t)=0,
$$

where the dot means time derivative. Usually, these coupled equations are interpreted as a linear system of ordinary differential equations for $f_{a}(t)$ when the $h_{a}(t)$ components of the Hamiltonian are known [45-48,50]. Instead, we put forward here a different, inverse perspective, and consider them to be an algebraic system to be solved for the $h_{a}(t)$, when the $f_{a}(t)$ are given. Definin the $N \times N$ matrix $\mathcal{A}$ as

$$
\mathcal{A}_{a b}(t)=\frac{1}{i \hbar} \sum_{c=1}^{N} \alpha_{a b c} f_{c}(t),
$$

Eq. (8) can be written as

$$
\begin{gathered}
\dot{f}_{a}(t)=\sum_{b=1}^{N} \mathcal{A}_{a b}(t) h_{b}(t), \\
\text { or }|\dot{\mathbf{f}}\rangle=\mathcal{A}|\mathbf{h}\rangle,
\end{gathered}
$$

where the kets are define in terms of the components of each generator, for example,

$$
|\mathbf{f}\rangle=\left(\begin{array}{c}
f_{1} \\
f_{2} \\
\cdots \\
f_{N}
\end{array}\right) .
$$

In this vector space, we may naturally refer to $|\mathbf{h}\rangle$ and $|\mathbf{f}\rangle$ as the Hamiltonian and the invariant, respectively. Even though the context should avoid any confusion with the vectors $|\Psi(t)\rangle$ define before in the state-vector space, the difference is nevertheless emphasized by the boldface notation. There are many Hamiltonians for a given invariant [49] and we cannot generally invert Eq. (11) as $|\mathbf{h}\rangle=\mathcal{A}^{-1}|\dot{\mathbf{f}}\rangle$ to get $|\mathbf{h}\rangle$. This means that $\operatorname{det}(\mathcal{A})=0$, so at least one of the eigenvalues $a^{(i)}(t)$ of the $\mathcal{A}$ matrix vanishes. To fin a valid $|\mathbf{h}\rangle$ in this case, one may use Gauss elimination, as shown in the Appendix. Alternatively, we shall follow here a more compact and intuitive operational approach. The $\mathcal{A}$ matrix may be generally non-Hermitian. It has $N$ right eigenvectors $\left\{\left|\mathbf{a}^{(i)}(t)\right\rangle\right\}, i=1,2, \ldots, N[17,51]$,

$$
\mathcal{A}(t)\left|\mathbf{a}^{(i)}(t)\right\rangle=a^{(i)}(t)\left|\mathbf{a}^{(i)}(t)\right\rangle,
$$

and biorthonormal partners $\left\{\left|\hat{\mathbf{a}}^{(i)}(t)\right\rangle\right\}$,

$$
\mathcal{A}^{\dagger}(t)\left|\hat{\mathbf{a}}^{(i)}(t)\right\rangle=\left(a^{(i)}(t)\right)^{*}\left|\hat{\mathbf{a}}^{(i)}(t)\right\rangle,
$$

where the asterisk means complex conjugate and the dagger denotes the adjoint. These eigenvectors are normalized as

$$
\left\langle\hat{\mathbf{a}}^{(i)}(t) \mid \mathbf{a}^{(j)}(t)\right\rangle=\delta_{i j},
$$

where bras are define as $\langle\mathbf{a}|=\left(a_{1}^{*}, a_{2}^{*}, \ldots, a_{N}^{*}\right)$, and the scalar product as $\langle\mathbf{a} \mid \mathbf{b}\rangle=a_{1}^{*} b_{1}+a_{2}^{*} b_{2}+\cdots+a_{N}^{*} b_{N}$. They satisfy closure relations

$$
\sum_{i=1}^{N}\left|\hat{\mathbf{a}}^{(i)}(t)\right\rangle\left\langle\mathbf{a}^{(i)}(t)\left|=\sum_{i=1}^{N}\right| \mathbf{a}^{(i)}(t)\right\rangle\left\langle\hat{\mathbf{a}}^{(i)}(t)\right|=\mathbb{I}_{N} .
$$

We can thus write the operator $\mathcal{A}(t)$ and its adjoint as

$$
\begin{gathered}
\mathcal{A}(t)=\sum_{i=1}^{N}\left|\mathbf{a}^{(i)}(t)\right\rangle a^{(i)}(t)\left\langle\hat{\mathbf{a}}^{(i)}(t)\right|, \\
\mathcal{A}^{\dagger}(t)=\sum_{i=1}^{N}\left|\hat{\mathbf{a}}^{(i)}(t)\right\rangle\left(a^{(i)}(t)\right)^{*}\left\langle\mathbf{a}^{(i)}(t)\right| .
\end{gathered}
$$

Let us defin the null-subspace projector $\mathcal{Q}$ of $\mathcal{A}$ associated with the $a^{(i)}(t)=0$ eigenvalue as $\mathcal{Q}=\sum_{i=1}^{Q}\left|\mathbf{a}^{(i)}(t)\right\rangle\left\langle\hat{\mathbf{a}}^{(i)}(t)\right|$, and the complementary projector $\mathcal{P}=\sum_{i=1}^{P}\left|\mathbf{a}^{(i)}(t)\right\rangle\left\langle\hat{\mathbf{a}}^{(i)}(t)\right|$. We have that $\mathcal{P}+\mathcal{Q}=\mathbb{I}_{N}$ and $P+Q=N$. Note as well that $\mathcal{P}$ and $\mathcal{Q}$ commute with $\mathcal{A}$ and the relations $\mathcal{P}^{2}=\mathcal{P}, \mathcal{Q}^{2}=\mathcal{Q}$, and $\mathcal{Q A}=0$. To solve $|\dot{\mathbf{f}}\rangle=\mathcal{A}|\mathbf{h}\rangle$ for $|\mathbf{h}\rangle$, we use Eq. (17) and project it firs onto the $\mathcal{P}$ subspace,

$$
\begin{aligned}
\mathcal{P}|\dot{\mathbf{f}}\rangle & =\sum_{i=1}^{N} \sum_{j=1}^{P}\left|\mathbf{a}^{(j)}\right\rangle\left\langle\hat{\mathbf{a}}^{(j)} \mid \mathbf{a}^{(i)}\right\rangle a^{(i)}\left\langle\hat{\mathbf{a}}^{(i)} \mid \mathbf{h}\right\rangle \\
& =\sum_{j=1}^{P}\left|\mathbf{a}^{(j)}\right\rangle a^{(j)}\left\langle\hat{\mathbf{a}}^{(j)} \mid \mathbf{h}\right\rangle .
\end{aligned}
$$

Since here all $a^{(j)}(t) \neq 0$, we can invert the expression,

$$
\sum_{i=1}^{P}\left|\mathbf{a}^{(i)}\right\rangle a^{(i)^{-1}}\left\langle\hat{\mathbf{a}}^{(i)} \mid \dot{\mathbf{f}}\right\rangle=\sum_{i=1}^{P}\left|\mathbf{a}^{(i)}\right\rangle\left\langle\hat{\mathbf{a}}^{(i)} \mid \mathbf{h}\right\rangle,
$$

so the $\mathcal{P}$ part of the solution is given by

$$
\mathcal{P}|\mathbf{h}\rangle=\mathcal{B}|\dot{\mathbf{f}}\rangle,
$$

where $\mathcal{B}=\mathcal{P B P}=\mathcal{P B}=\mathcal{B P}=\sum_{i=1}^{P}\left|\mathbf{a}^{(i)}\right\rangle a^{(i)}{ }^{-1}\left\langle\hat{\mathbf{a}}^{(i)}\right|$ is a pseudoinverse matrix of $\mathcal{A}$, as $\mathcal{A B} \mathcal{A}=\mathcal{A}$. This relation implies $P$ equations for the $\left\{h_{j}\right\},\left\{f_{j}\right\}$, and $\left\{\dot{f}_{j}\right\}$. If, instead, $|\dot{\mathbf{f}}\rangle=\mathcal{A}|\mathbf{h}\rangle$ is projected onto the null subspace, we get

$$
\mathcal{Q}|\dot{\mathbf{f}}\rangle=\sum_{j=1}^{Q}\left|\mathbf{a}^{(j)}\right\rangle a^{(j)}\left\langle\hat{\mathbf{a}}^{(j)} \mid \mathbf{h}\right\rangle=0,
$$

because now all $a^{(j)}(t)=0$. This relation implies the existence of multiple solutions for $|\mathbf{h}\rangle$, and $Q$ conditions $\left\langle\hat{\mathbf{a}}^{(j)} \mid \hat{\mathbf{f}}\right\rangle=0$ which involve $\left\{f_{i}\right\}$ and their time derivatives. We can add any arbitrary part $\mathcal{Q}|\mathbf{h}\rangle$ to Eq. (20) so that all Hamiltonians of the form

$$
|\mathbf{h}\rangle=\mathcal{B}|\dot{\mathbf{f}}\rangle+\mathcal{Q}|\mathbf{h}\rangle,
$$


where $\mathcal{Q}|\mathbf{h}\rangle$ is a completely arbitrary vector in the null subspace, are thus compatible with the invariant $I$. This is one of the fundamental equations of this paper. Due to the freedom to choose $\mathcal{Q}|\mathbf{h}\rangle$ (we may construct it as $\mathcal{Q}|\mathbf{b}\rangle$, where $|\mathbf{b}\rangle$ is arbitrary), we can change the Hamiltonian to make it realizable. In addition, the invariant itself may be modified

When inverse engineering shortcuts to adiabaticity, the Hamiltonian is usually given at initial and fina times. In general, the invariant $I$ [equivalently $|\mathbf{f}(t)\rangle$ ] is chosen to drive, through its eigenvectors, the initial states of the Hamiltonian $H(0)$ to the states of the fina $H\left(t_{f}\right)[1,7,44]$. This is ensured by imposing, at the boundary times $t_{b}=0, t_{f}$, the "frictionless conditions" $\left[H\left(t_{b}\right), I\left(t_{b}\right)\right]=0[1]$. For Eqs. (1) and (7), these boundary conditions can be reformulated as

$$
\sum_{a, b, c}^{N} \alpha_{a b c} h_{b}\left(t_{b}\right) f_{c}\left(t_{b}\right) T_{a}=0
$$

Since the $T_{a}$ generators are independent, the coefficient must satisfy

$$
\sum_{b, c}^{N} \alpha_{a b c} h_{b}\left(t_{b}\right) f_{c}\left(t_{b}\right)=0, a=1, \ldots, N, t_{b}=0, t_{f},
$$

or, more compactly,

$$
\mathcal{A}\left(t_{b}\right)\left|\mathbf{h}\left(t_{b}\right)\right\rangle=0, t_{b}=0, t_{f},
$$

which is a second fundamental result. Note that the choice of $\mathcal{Q}|\mathbf{h}\rangle$ does not affect this condition, but $|\mathbf{f}\rangle$ must be chosen to fulfil it. At the boundary times, Eq. (25) imposes $N$ conditions, and, if the $N$ values of the $\left\{h_{j}\left(t_{b}\right)\right\}$ are given, the $P+Q$ equations in Eqs. (20) and (21) will fi the values of $\left\{f_{j}\left(t_{b}\right)\right\}$ and $\left\{\dot{f}_{j}\left(t_{b}\right)\right\}$. At intermediate times, the Hamiltonian and invariant components can be designed subjected to the $N$ equations in Eqs. (20) and (21) and to the boundary conditions. This leaves open different inverse engineering possibilities: in general, the Hamiltonian is firs fi ed partially, i.e., imposing the time dependence (or vanishing) of some $r<N$ components. Fixing the invariant time dependence consistently with the boundary conditions and the imposed Hamiltonian constraints finall leads to equations that give the form of the remaining $N-r$ Hamiltonian components. The following sections illustrate these steps and concepts explicitly.

\section{EXAMPLE 1: SU(2) LIE ALGEBRA}

Let us consider the $\mathrm{SU}(2)$ algebra $(N=3)$ spanned by $\left\{T_{1}, T_{2}, T_{3}\right\}$ with commutation relations

$$
\left[T_{1}, T_{2}\right]=i T_{3},\left[T_{2}, T_{3}\right]=i T_{1},\left[T_{3}, T_{1}\right]=i T_{2} .
$$

Equation (10) becomes

$$
\left(\begin{array}{l}
\dot{f}_{1} \\
\dot{f}_{2} \\
\dot{f}_{3}
\end{array}\right)=\underbrace{\frac{1}{\hbar}\left(\begin{array}{ccc}
0 & f_{3} & -f_{2} \\
-f_{3} & 0 & f_{1} \\
f_{2} & -f_{1} & 0
\end{array}\right)}_{=\mathcal{A}}\left(\begin{array}{l}
h_{1} \\
h_{2} \\
h_{3}
\end{array}\right) .
$$

As $\mathcal{A}=-\mathcal{A}^{\dagger}$ is a real antisymmetric matrix with odd dimensionality, the eigenvalues are conjugate pure imaginary pairs, and zero, whereas left and right eigenvectors are equal. Explicitly, the eigenvalues are $a^{(0)}=0, a^{(1)}=-i \sqrt{\gamma} / \hbar$, and $a^{(2)}=i \sqrt{\gamma} / \hbar$ (we have shifted down the superscripts by one with respect to the general formalism, here $j=0,1,2$, so that the zero corresponds to the zero eigenvalue), with

$$
\gamma=f_{1}^{2}+f_{2}^{2}+f_{3}^{2}
$$

and the eigenvectors

$$
\begin{aligned}
& \left|a^{(0)}\right\rangle=\left|\hat{a}^{(0)}\right\rangle=\frac{1}{w_{0}}\left(\begin{array}{c}
\frac{f_{1}}{f_{3}} \\
\frac{f_{2}}{f_{3}} \\
1
\end{array}\right), \\
& \left|a^{(1)}\right\rangle=\left|\hat{a}^{(1)}\right\rangle=\frac{1}{w_{1}}\left(\begin{array}{c}
\frac{-f_{1} f_{3}-i f_{2} \sqrt{\gamma}}{\beta-\gamma} \\
\frac{-f_{1}^{2}-f_{3}^{2}}{f_{2} f_{3}+i f_{1} \sqrt{\gamma}} \\
1
\end{array}\right), \\
& \left|a^{(2)}\right\rangle=\left|\hat{a}^{(2)}\right\rangle=\frac{1}{w_{2}}\left(\begin{array}{c}
\frac{-f_{1} f_{3}+i f_{2} \sqrt{\gamma}}{\beta-\gamma} \\
\frac{-f_{1}^{2}-f_{3}^{2}}{f_{2} f_{3}-i f_{1} \sqrt{\gamma}} \\
1
\end{array}\right),
\end{aligned}
$$

where $w_{2}=w_{1}=\sqrt{2 \gamma /(\beta-\gamma)}, w_{0}=\sqrt{\gamma / f_{3}^{2}}$, and

$$
\beta=2\left(f_{1}^{2}+f_{2}^{2}\right)+f_{3}^{2} \text {. }
$$

The $\mathcal{P}$ and $\mathcal{Q}$ projectors are

$$
\mathcal{P}=\mathbb{I}_{3}-\mathcal{Q}, \quad \mathcal{Q}=\frac{1}{\gamma}\left(\begin{array}{ccc}
f_{1}^{2} & f_{1} f_{2} & f_{1} f_{3} \\
f_{1} f_{2} & f_{2}^{2} & f_{2} f_{3} \\
f_{1} f_{3} & f_{2} f_{3} & f_{3}^{2}
\end{array}\right),
$$

whereas the pseudoinverse matrix $\mathcal{B}$ [see Eq. (20)] is

$$
\mathcal{B}=\frac{\hbar}{\gamma}\left(\begin{array}{ccc}
0 & -f_{3} & f_{2} \\
f_{3} & 0 & -f_{1} \\
-f_{2} & f_{1} & 0
\end{array}\right)
$$

From Eq. (21), we get the condition that the system has infinit solutions,

$$
\left(\begin{array}{l}
\frac{f_{1}\left(f_{1} \dot{f}_{1}+f_{2} \dot{f}_{2}+f_{3} \dot{f}_{3}\right)}{f_{1}^{2}+f_{2}^{2}+f_{3}^{2}} \\
\frac{f_{2}\left(f_{1} \dot{f}_{1}+f_{2} \dot{f}_{2}+f_{3} \dot{f}_{3}\right)}{f_{1}^{2}+f_{2}^{2}+f_{3}^{2}} \\
\frac{f_{3}\left(f_{1} \dot{f}_{1}+f_{2} \dot{f}_{2}+f_{3} \dot{f}_{3}\right)}{f_{1}^{2}+f_{2}^{2}+f_{3}^{2}}
\end{array}\right)=0 .
$$

As there is only one independent eigenvector for the null subspace, $Q=1$, this is solved by a single condition,

$$
f_{1} \dot{f}_{1}+f_{2} \dot{f}_{2}+f_{3} \dot{f}_{3}=0,
$$

i.e., $\gamma=$ const, and the $f_{i}$ cannot be arbitrary independent functions. Equation (20) becomes

$$
\mathcal{P}\left(\begin{array}{l}
h_{1} \\
h_{2} \\
h_{3}
\end{array}\right)=\frac{\hbar}{\gamma}\left(\begin{array}{c}
f_{2} \dot{f}_{3}-f_{3} \dot{f}_{2} \\
-f_{1} \dot{f}_{3}-f_{3} \dot{f}_{1} \\
f_{1} \dot{f}_{2}-f_{2} \dot{f}_{1}
\end{array}\right),
$$

and the general solution (22) takes the form

$$
\left(\begin{array}{l}
h_{1} \\
h_{2} \\
h_{3}
\end{array}\right)=\frac{\hbar}{\gamma}\left(\begin{array}{c}
-\dot{f}_{2} f_{3}+f_{2} \dot{f}_{3}+f_{1}\langle\mathbf{h} \mid \mathbf{f}\rangle / \hbar \\
\dot{f}_{1} f_{3}-f_{1} \dot{f}_{3}+f_{2}\langle\mathbf{h} \mid \mathbf{f}\rangle / \hbar \\
-\dot{f}_{1} f_{2}+f_{1} \dot{f}_{2}+f_{3}\langle\mathbf{h} \mid \mathbf{f}\rangle / \hbar
\end{array}\right) .
$$



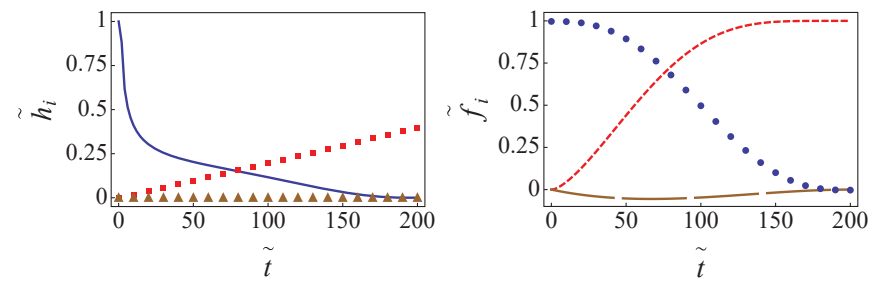

FIG. 1. (Color online) Hamiltonian (left) and invariant (right) coefficient vs time. The imposed functions (symbols) are $h_{1}(t)$ (red squares), $h_{2}(t)$ (brown triangles), and $f_{3}(t)$ (blue circles). The derived functions (lines) are $h_{3}(t)$ (blue solid line), $f_{1}(t)$ (red short-dashed line), and $f_{2}(t)$ (brown long-dashed line). Parameter values: $\tilde{h}_{1}\left(t_{f}\right)=0.4, \tilde{h}_{3}(0)=1$, and $\tilde{t}_{f}=200$.

Using Eq. (34), this gives the compact result

$$
h_{i}=-\hbar \mathcal{E}_{i j k} \frac{\dot{f_{j}}}{f_{k}}+\frac{f_{i}}{f_{k}} h_{k},
$$

with all indices $i, j, k$ different. $\mathcal{E}_{i j k}$ is the Levy-Civita symbol [ 1 for even permutations of (123) and -1 for odd permutations] and $h_{k}(t)$ is considered a free function chosen for convenience. If we want to impose, for example, that one of the components of the Hamiltonian is zero, then we take that component to be $h_{k}$.

\section{A. Two-level system}

To be more specific let us consider the following representation useful to describe, for example, a two-level system in an external driving field

$T_{1}=\frac{1}{2}\left(\begin{array}{ll}0 & 1 \\ 1 & 0\end{array}\right), \quad T_{2}=\frac{1}{2}\left(\begin{array}{cc}0 & -i \\ i & 0\end{array}\right), \quad T_{3}=\frac{1}{2}\left(\begin{array}{cc}1 & 0 \\ 0 & -1\end{array}\right)$.

We set as initial and fina constraints the following Hamiltonians:

$$
\begin{gathered}
H(0)=h_{1}(0) T_{1}+h_{3}(0) T_{3}, \\
H\left(t_{f}\right)=h_{1}\left(t_{f}\right) T_{1}+h_{3}\left(t_{f}\right) T_{3},
\end{gathered}
$$

but, in general, $H(t)=h_{1}(t) T_{1}+h_{2}(t) T_{2}+h_{3}(t) T_{3}$. The frictionless conditions (25) for SU(2) read

$$
f_{i}\left(t_{b}\right) h_{j}\left(t_{b}\right)-f_{j}\left(t_{b}\right) h_{i}\left(t_{b}\right)=0, i>j .
$$

Our aim is to fin $H(t)$ so that the ground and excited states of $H(0)$ become ground and excited states of $H\left(t_{f}\right)$ in an arbitrary time $t_{f}$, up to phase factors, in such a way that $h_{2}(t)=0 \forall t$. This condition is motivated by the difficult to implement $T_{2}$ in some systems [16]. Choosing $(i, j, k)=(1,3,2)$ in Eq. (37), with $h_{2}(t)=0$ and using $\gamma=$ const $=c_{1}$, we can express $f_{2}$ and $f_{1}$ in terms of $f_{3}$,

$$
\begin{aligned}
& f_{2}=\frac{\hbar \dot{f}_{3}}{h_{1}}, \\
& f_{1}=\sqrt{c_{1}-f_{3}^{2}-\frac{\hbar^{2} \dot{f}_{3}^{2}}{h_{1}^{2}} .}
\end{aligned}
$$
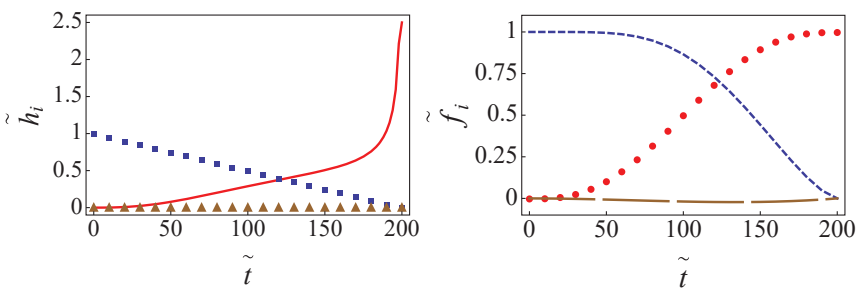

FIG. 2. (Color online) Hamiltonian (left) and invariant (right) coefficient vs time. The imposed functions (symbols) are $h_{2}(t)$ (brown triangles), $h_{3}(t)$ (blue squares), and $f_{1}(t)$ (red circles). The derived functions (lines) are $h_{1}(t)$ (red solid line), $f_{2}(t)$ (brown long-dashed line), and $f_{3}(t)$ (blue short-dashed line). Parameter values: $\tilde{h}_{3}(0)=1, \tilde{h}_{1}\left(t_{f}\right)=2.5$, and $\tilde{t}_{f}=200$.

Substituting this in the other equation of Eq. (37), with $(i, j, k)=(3,1,2)$,

$$
\ddot{f}_{3}-\frac{\dot{h}_{1}}{h_{1}} \dot{f}_{3}+\frac{h_{1}}{\hbar^{2}}\left(f_{3} h_{1}-h_{3} \sqrt{c_{1}-f_{3}^{2}-\frac{\hbar^{2} \dot{f}_{3}^{2}}{h_{1}^{2}}}\right)=0 .
$$

The frictionless conditions (41) for this case impose $f_{2}\left(t_{b}\right)=0$ and $h_{3}\left(t_{b}\right) / h_{1}\left(t_{b}\right)=f_{3}\left(t_{b}\right) / f_{1}\left(t_{b}\right)$ or, equivalently,

$$
f_{3}\left(t_{b}\right)=h_{3}\left(t_{b}\right) \sqrt{\frac{c_{1}}{h_{1}^{2}\left(t_{b}\right)+h_{3}^{2}\left(t_{b}\right)}}, \quad \dot{f}_{3}\left(t_{b}\right)=0 .
$$

In addition, from Eq. (43) at the boundary times $t_{b}$,

$$
\ddot{f}_{3}\left(t_{b}\right)=0 \text {. }
$$

An example of a possible Hamiltonian engineering strategy is to firs impose, in addition to $h_{2}(t)=0$, the form of $h_{1}(t)$ also. We then interpolate $f_{3}(t)$ (with a simple polynomial or following some more sophisticated approach, e.g., to optimize some variable) satisfying the boundary conditions (44) and (45) at the boundary times $t_{b}$, and solve for $h_{3}(t)$ in Eq. (43). In the example of Fig. 1, the initial ground state of Eq. (39) with $h_{1}(0)=0$ is placed at the north pole of the Bloch sphere and it is driven to the equator of the sphere ending as the ground state of Eq. (40), with $h_{3}\left(t_{f}\right)=0 . h_{3}(t)$ is deduced assuming $h_{1}(t)=$ $h_{1}\left(t_{f}\right) t / t_{f}$ and $f_{3}(t)=\sum_{i=0}^{5} b_{i} t^{i}$, where the $b_{i}$ coefficient are deduced from the boundary conditions (44) and (45). We set $c_{1}=h_{1}^{2}(0)+h_{3}^{2}(0)$ so that $H(0)=I(0)$. In this and the rest of the figures we plot dimensionless variables $\tilde{t}=t \sqrt{c_{1}} / \hbar$ and $\tilde{E}=E / \sqrt{c_{1}}$, and the invariant has been set with dimensions of energy. To fin solutions with real functions, the condition

$$
f_{3}^{2}+\frac{\hbar^{2} \dot{f}_{3}^{2}}{h_{1}^{2}} \leqslant c_{1} \forall t
$$

must be satisfied see Eqs. (42) and (43). This sets a minimum fina time $t_{f, m}$ that depends on the constant $c_{1}$ and the ansatz to interpolate $f_{3}(t)$. The (dimensionless) minimum time for the parameter values considered in Fig. 1 is $\tilde{t}_{f, m} \sim 165$.

Note that Eq. (43) is an algebraic equation for $h_{3}(t)$ and a differential equation for $h_{1}(t)$. Other option is to set $h_{3}(t)$ firs and deduce $h_{1}(t)$ from Eq. (43), which is now a differential equation for this variable. To solve instead for $h_{1}(t)$ algebraically, we may engineer $f_{1}(t)$ rather than $f_{3}(t)$, with an equation of the form of Eq. (43) that has the indices 1 and 3 swapped; see Fig. 2. 


\section{EXAMPLE 2: U3S3}

As a second example, consider the four-dimensional $(N=$ 4) Lie algebra U3S3 [52], with basis $\left\{T_{1}, T_{2}, T_{3}, T_{4}\right\}$, where $\left\{T_{1}, T_{2}, T_{3}\right\}$ span a $\mathrm{SU}(2)$ subalgebra [see Eq. (26)], whereas the only nonvanishing commutators of $T_{4}$ are

$$
\left[T_{4}, T_{1}\right]=i T_{2},\left[T_{2}, T_{4}\right]=i T_{1} .
$$

Since $T_{4}-T_{3}$ commutes with any member of the algebra (it is an invariant in a Lie-algebraic sense), this combination might appear as the natural fourth basis generator instead of $T_{4}$. However, the use of $T_{4}$ is physically motivated by its natural occurrence in the system we shall deal with, namely, two interacting bosons in two wells [53]. The $\mathcal{A}$ matrix for our basis choice is

$$
\mathcal{A}=\frac{1}{\hbar}\left(\begin{array}{cccc}
0 & -f_{3} & f_{2} & f_{2} \\
f_{3} & 0 & -f_{1} & f_{1} \\
-f_{2} & f_{1} & 0 & 0 \\
-f_{2} & f_{1} & 0 & 0
\end{array}\right),
$$

with eigenvalues $a^{(0)}=a^{(1)}=0, a^{(2)}=-i \beta / \hbar$, and $a^{(3)}=$ $i \beta / \hbar$. The left and right eigenvectors are

$$
\begin{aligned}
& \left|a^{(0)}\right\rangle=\left|\hat{a}^{(0)}\right\rangle=\frac{1}{\sqrt{\gamma}}\left(\begin{array}{c}
f_{1} \\
f_{2} \\
0 \\
f_{3}
\end{array}\right), \\
& \left|a^{(1)}\right\rangle=\left|\hat{a}^{(1)}\right\rangle=\frac{1}{\sqrt{\gamma \beta}}\left(\begin{array}{c}
f_{1} f_{3} \\
f_{2} f_{3} \\
\gamma \\
\beta-\gamma
\end{array}\right), \\
& \operatorname{brk}\left|a^{(2)}\right\rangle=\left|\hat{a}^{(2)}\right\rangle=\frac{1}{\sqrt{2 \beta}}\left(\begin{array}{c}
\frac{-f_{1} f_{3}+i f_{2} \sqrt{\beta}}{\beta-\gamma} \\
\frac{2 f_{1}^{2}+f_{3}^{2}}{-f_{2} f_{3}+i f_{1} \sqrt{\beta}} \\
\sqrt{\beta-\gamma} \\
\sqrt{\beta-\gamma}
\end{array}\right) \text {, } \\
& \left|a^{(3)}\right\rangle=\left|\hat{a}^{(3)}\right\rangle=\frac{1}{\sqrt{2 \beta}}\left(\begin{array}{c}
\frac{-f_{1} f_{3}-i f_{2} \sqrt{\beta}}{\beta-\gamma} \\
\frac{-f_{2} f_{3}+i f_{1} \sqrt{\beta}}{\beta-\gamma} \\
\sqrt{\beta-\gamma} \\
\sqrt{\beta-\gamma}
\end{array}\right),
\end{aligned}
$$

where $\gamma$ and $\beta$ are define as before; see Eqs. (28) and (30). Note that here, unlike the previous example the zero eigenvalue is degenerate, so the null subspace has dimension $Q=2$. Equation (21) now sets two conditions: one is the same condition as for SU(2), $\dot{f}_{1} f_{1}+\dot{f}_{2} f_{2}+\dot{f}_{3} f_{3}=0$, and the other one is $\dot{f}_{3}=\dot{f}_{4}$, so the system $|\dot{\mathbf{f}}\rangle=\mathcal{A}|\mathbf{h}\rangle$ has solution for $|\mathbf{h}\rangle$ if

$$
\begin{gathered}
f_{1}^{2}+f_{2}^{2}+f_{3}^{2}=c_{1}, \\
f_{4}=f_{3}+c_{2},
\end{gathered}
$$

with $c_{1}$ and $c_{2}$ constants (there are two independent $f_{i}$ ). We use now Eq. (22) as in the previous example to get

$$
\begin{aligned}
& h_{1}=\frac{\hbar\left(\dot{f}_{1} f_{1}+\dot{f}_{2} f_{2}\right)+f_{1} f_{3} h_{2}}{f_{2} f_{3}}, \\
& h_{4}=\frac{\hbar \dot{f}_{1}+f_{3} h_{2}-f_{2} h_{3}}{f_{2}} .
\end{aligned}
$$

Due to degeneracy of the null eigenvalue, there are two free $h_{i}$, and we have chosen them to be $h_{2}$ and $h_{3}$ here. The frictionless conditions $\mathcal{A}\left(t_{b}\right)\left|\mathbf{h}\left(t_{b}\right)\right\rangle=0$ become explicitly

$$
\begin{aligned}
-f_{3}\left(t_{b}\right) h_{2}\left(t_{b}\right)+f_{2}\left(t_{b}\right) h_{3}\left(t_{b}\right)+f_{2}\left(t_{b}\right) h_{4}\left(t_{b}\right) & =0, \\
f_{3}\left(t_{b}\right) h_{1}\left(t_{b}\right)-f_{1}\left(t_{b}\right) h_{3}\left(t_{b}\right)+f_{1}\left(t_{b}\right) h_{4}\left(t_{b}\right) & =0, \\
- & f_{2}\left(t_{b}\right) h_{1}\left(t_{b}\right)+f_{1}\left(t_{b}\right) h_{2}\left(t_{b}\right)=0 .
\end{aligned}
$$

\section{A. Two interacting bosons in a double well}

An interacting boson gas in a lattice potential can be described by the Bose-Hubbard model [54,55]. For two particles in two wells, the Hamiltonian in the occupation number basis $\left\{\left|n_{\text {left }}, n_{\text {right }}\right\rangle\right\}:|2,0\rangle=\left(\begin{array}{l}1 \\ 0 \\ 0\end{array}\right),|1,1\rangle=\left(\begin{array}{l}0 \\ 1 \\ 0\end{array}\right)$, and $|0,2\rangle=\left(\begin{array}{l}0 \\ 0 \\ 1\end{array}\right)$, is given by [53]

$$
H_{0}=\left(\begin{array}{ccc}
U & -\sqrt{2} J & 0 \\
-\sqrt{2} J & 0 & -\sqrt{2} J \\
0 & -\sqrt{2} J & U
\end{array}\right)=U T_{4}-4 J T_{1},
$$

where $U$ gives the interparticle interaction energy and $J$ is a hopping constant, assumed to be controllable functions of time, and

$$
T_{1}=\frac{1}{2 \sqrt{2}}\left(\begin{array}{lll}
0 & 1 & 0 \\
1 & 0 & 1 \\
0 & 1 & 0
\end{array}\right), \quad T_{4}=\left(\begin{array}{lll}
1 & 0 & 0 \\
0 & 0 & 0 \\
0 & 0 & 1
\end{array}\right) .
$$

We may close the algebra with two additional generators,

$$
T_{2}=\frac{1}{2 \sqrt{2}}\left(\begin{array}{ccc}
0 & -i & 0 \\
i & 0 & i \\
0 & -i & 0
\end{array}\right), T_{3}=\frac{1}{4}\left(\begin{array}{ccc}
1 & 0 & 1 \\
0 & -2 & 0 \\
1 & 0 & 1
\end{array}\right),
$$

where the $T_{a}$ satisfy the commutation relations given by Eqs. (26) and (47). Let us consider that at initial and fina times, the Hamiltonian of our system is

$$
\begin{gathered}
H(0)=h_{1}(0) T_{1}+h_{4}(0) T_{4}, \\
H\left(t_{f}\right)=h_{1}\left(t_{f}\right) T_{1}+h_{4}\left(t_{f}\right) T_{4},
\end{gathered}
$$

and we want to drive without fina excitation the ground state of $H(0)$ to $H\left(t_{f}\right)$. In [53], the shortcut-to-adiabaticity method that is followed requires the addition of the "counterdiabatic term" proportional to $T_{2}$, which is difficul to implement [53]. $T_{3}$ is also problematic, so we shall engineer the Hamiltonian with Eq. (52) imposing $h_{2}(t)=h_{3}(t)=0 \forall t$. Then, using Eq. (50),

$$
\begin{aligned}
& f_{2}=\frac{\hbar \dot{f}_{1}}{h_{4}}, \\
& f_{3}=\sqrt{c_{1}-f_{1}^{2}-\frac{\hbar^{2} \dot{f}_{1}^{2}}{h_{4}^{2}}},
\end{aligned}
$$



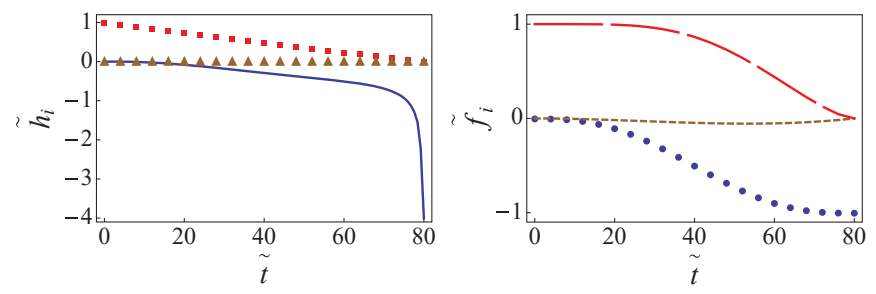

FIG. 3. (Color online) Hamiltonian (left) and invariant (right) coefficient vs time. The imposed functions (symbols) are $h_{2}(t)$ (brown triangles), $h_{3}(t)$, (brown triangles), $h_{4}(t)$ (red squares), and $f_{1}(t)$ (blue circles). The derived functions (lines) are $h_{1}(t)$ (blue solid line), $f_{2}(t)$ (brown, short-dashed line), $f_{3}(t)$ (red long-dashed line), and $f_{4}(t)$ (red long-dashed line). Parameter values: $\tilde{h}_{1}\left(t_{f}\right)=-4$, $\tilde{h}_{4}(0)=1$, and $\tilde{t}_{f}=80$.

where $f_{1}$ satisfie

$$
\ddot{f}_{1}-\frac{\dot{h}_{4}}{h_{4}} \dot{f}_{1}+\frac{h_{4}}{\hbar^{2}}\left(f_{1} h_{4}-h_{1} \sqrt{c_{1}-f_{1}^{2}-\frac{\hbar^{2} \dot{f}_{1}^{2}}{h_{4}^{2}}}\right)=0 .
$$

Additionally, $f_{4}$ is given by Eq. (51). The frictionless conditions (53) for $h_{2}\left(t_{b}\right)=h_{3}\left(t_{b}\right)=0$ are

$$
f_{1}\left(t_{b}\right)=h_{1}\left(t_{b}\right) \sqrt{\frac{c_{1}}{h_{1}^{2}\left(t_{b}\right)+h_{4}^{2}\left(t_{b}\right)}}, \quad \dot{f}_{1}\left(t_{b}\right)=0,
$$

and from Eq. (60), at the boundary times $t_{b}$,

$$
\ddot{f}_{1}\left(t_{b}\right)=0 \text {. }
$$

Assuming that $h_{4}(t)$ is imposed, Eq. (60) sets $h_{1}(t)$ to drive the initial ground state of $H(0)$ to $H\left(t_{f}\right)$ without undesired terms. In Fig. 3, the Hamiltonian and the invariant components are plotted for a frictionless Mott-insulator-to-superflui transition $[53,56]$. The initial ground state of Eq. (57) with $h_{1}(0)=0$ corresponds to a Mott insulator and it evolves into the superflui ground state of Eq. (58) with $h_{4}\left(t_{f}\right)=0$. We assume a linear variation of $h_{4}(t)=h_{4}(0)\left(1-t / t_{f}\right)$ and $f_{1}(t)=\sum_{i=0}^{5} \bar{b}_{i} t^{i}$, where the $\bar{b}_{i}$ are deduced from the boundary conditions (61) and (62), $c_{1}=h_{1}^{2}(0)+h_{4}^{2}(0)$, and $c_{2}=0$.

Similarly to the previous example, if we impose the form of $h_{1}(t)$ instead of $h_{4}, h_{4}(t)$ can be deduced algebraically from Eq. (60), replacing $f_{1}$ by $f_{3}$ and then swapping 4 and 1 .

\section{OUTLOOK}

We have worked out a framework to engineer timedependent Hamiltonians and speed up adiabatic processes making use of dynamical invariants and dynamical algebras. This is particularly useful to fin shortcuts free from Hamiltonian terms that are difficul to implement in practice. Explicit construction formulas allow us to fi some components of the Hamiltonian, to make them zero, for example, and get the time dependence of the remaining components.

This work should be distinguished from a related method presented in a companion paper [56]. Both approaches share the use of Lie-algebraic methods and the aim of constructing shortcuts. However, the approach presented here is a systematic bottom-up inverse engineering method based on the relation between Hamiltonian and dynamical invariants.
Instead, in [56], the dynamical invariants do not play an explicit role. The starting point for the approach in [56] is an existing, known shortcut; then, unitary transformations are carried out to generate alternative (feasible or more convenient) shortcuts, as in [41]. The connection between the two approaches is left for a separate study.

We also mention some fundamental questions worth investigating: The type and structure of the algebra is expected to determine the inverse engineering possibilities and limitations, which are still little known. In particular, the role of Liealgebraic invariants (in contrast to dynamical invariants) or subalgebras should be examined [57], and quantum control theory [58], which overlaps in part with our objectives, may shed light on permissible or precluded operations. Adapting the current ideas to many-body systems is a further open question that may benefi from approaches based on restricting the action of the Hamiltonian to a subspace [31].

The emphasis here has been on the cancellation of undesired Hamiltonian terms, but other applications of the proposed Hamiltonian engineering are possible, for example, to optimize variables or robustness versus noise $[59,60]$, transient energy, and other relevant variables [9]. Finally, the formalism proposed may be extended to open systems governed by dynamical equations formulated by closed Lie algebras [61-64].

\section{ACKNOWLEDGMENTS}

We are grateful to K. Takahashi and R. Kosloff for stimulating discussions. We acknowledge funding by Grants No. IT472-10 and No. FIS2009-12773-C02-01, and the UPV/EHU Program No. UFI 11/55. E.T. is supported by the Basque Government postdoctoral program. S.M.-G. acknowledges support from a UPV/EHU fellowship.

\section{APPENDIX: GAUSS ELIMINATION}

A way to solve the system (11) for $|\mathbf{h}(t)\rangle$ is to use Gauss elimination. We consider explicitly the $\mathrm{SU}(2)$ group. The augmented matrix associated with the system in Eq. (27) is

$$
\left(\begin{array}{cccc}
-f_{3} & 0 & f_{1} & \hbar \dot{f}_{2} \\
0 & f_{3} & -f_{2} & \hbar \dot{f}_{1} \\
f_{2} & -f_{1} & 0 & \hbar \dot{f}_{3}
\end{array}\right)
$$

The essence of the method is to reduce the system to an equivalent one with the same solutions by applying elementary operations. These are the multiplication of a row by a nonzero scalar, the interchange of columns or rows, and the addition to a row of the multiple of a different one. In a firs step, $\left(f_{2} / f_{3}\right)$ times the firs row is added to the third one,

$$
\left(\begin{array}{cccc}
-f_{3} & 0 & f_{1} & \hbar \dot{f_{2}} \\
0 & f_{3} & -f_{2} & \hbar \dot{f_{1}} \\
0 & -f_{1} & \frac{f_{1} f_{2}}{f_{3}} & \frac{\hbar f_{2} \dot{f}_{2}}{f_{3}}+\hbar \dot{f_{3}}
\end{array}\right) .
$$

Finally, $\left(f_{1} / f_{3}\right)$ times the second row is added to the third, producing a lower triangular matrix, 


$$
\left(\begin{array}{cccc}
-f_{3} & 0 & f_{1} & \hbar \dot{f}_{2} \\
0 & f_{3} & -f_{2} & \hbar \dot{f}_{1} \\
0 & 0 & 0 & \frac{\hbar\left(f_{1} \dot{f}_{1}+f_{2} \dot{f}_{2}+f_{3} \dot{f}_{3}\right)}{f_{3}}
\end{array}\right) .
$$

This system is compatible and has infinit solutions if $f_{1} \dot{f}_{1}+$ $f_{2} \dot{f}_{2}+f_{3} \dot{f}_{3}=0$ or, equivalently, $f_{1}^{2}+f_{2}^{2}+f_{3}^{2}=c_{1}$. The solutions satisfy

$$
\begin{aligned}
& \hbar \dot{f}_{1}=f_{3} h_{2}-f_{2} h_{3}, \\
& \hbar \dot{f}_{2}=-f_{3} h_{1}+f_{1} h_{3},
\end{aligned}
$$

from which Eq. (37) follows.
[1] X. Chen, A. Ruschhaupt, S. Schmidt, A. del Campo, D. GuéryOdelin, and J. G. Muga, Phys. Rev. Lett. 104, 063002 (2010).

[2] D. Stefanatos, J. Ruths, and J.-S. Li, Phys. Rev. A 82, 063422 (2010).

[3] J. F. Schaff, P. Capuzzi, G. Labeyrie, and P. Vignolo, New J. Phys. 13, 113017 (2011).

[4] E. Torrontegui, Xi Chen, M. Modugno, A. Ruschhaupt, D. Guéry-Odelin, and J. G. Muga, Phys. Rev. A 85, 033605 (2012).

[5] A. Couvert, T. Kawalec, G. Reinaudi, and D. Guéry-Odelin, Europhys. Lett. 83, 13001 (2008).

[6] M. Murphy, L. Jiang, N. Khaneja, and T. Calarco, Phys. Rev. A 79, 020301(R) (2009).

[7] E. Torrontegui, S. Ibáñez, X. Chen, A. Ruschhaupt, D. GuéryOdelin, and J. G. Muga, Phys. Rev. A 83, 013415 (2011).

[8] E. Torrontegui, X. Chen, M. Modugno, S. Schmidt, A. Ruschhaupt, and J. G. Muga, New J. Phys. 14, 013031 (2012).

[9] X. Chen, E. Torrontegui, D. Stefanatos, J.-S. Li, and J. G. Muga, Phys. Rev. A 84, 043415 (2011).

[10] M. Palmero, E. Torrontegui, D. Guéry-Odelin, and J. G. Muga, Phys. Rev. A 88, 053423 (2013).

[11] M. Demirplak and S. A. Rice, J. Phys. Chem. 107, 9937 (2003).

[12] M. Demirplak and S. A. Rice, J. Phys. Chem. B 109, 6838 (2005).

[13] M. Demirplak and S. A. Rice, J. Chem. Phys. 129, 154111 (2008).

[14] M. V. Berry, J. Phys. A 42, 365303 (2009).

[15] X. Chen, I. Lizuain, A. Ruschhaupt, D. Guéry-Odelin, and J. G. Muga, Phys. Rev. Lett. 105, 123003 (2010).

[16] M. G. Bason, M. Viteau, N. Malossi, P. Huillery, E. Arimondo, D. Ciampini, R. Fazio, V. Giovannetti, R. Mannella, and O. Morsch, Nat. Phys. 8, 147 (2012).

[17] S. Ibáñez, S. Martínez-Garaot, X. Chen, E. Torrontegui, and J. G. Muga, Phys. Rev. A 84, 023415 (2011).

[18] Y. Ban, X. Chen, E. Y. Sherman, and J. G. Muga, Phys. Rev. Lett. 109, 206602 (2012).

[19] U. Hohenester, P. K. Rekdal, A. Borzi, and J. Schmiedmayer, Phys. Rev. A 75, 023602 (2007).

[20] J. Grond, J. Schmiedmayer, and U. Hohenester, Phys. Rev. A 79, 021603 (2009).

[21] J. Grond, G. von Winckel, J. Schmiedmayer, and U. Hohenester, Phys. Rev. A 80, 053625 (2009).

[22] S. Masuda and K. Nakamura, Proc. R. Soc. A 466, 1135 (2010).

[23] E. Torrontegui, S. Martínez-Garaot, A. Ruschhaupt, and J. G. Muga, Phys. Rev. A 86, 013601 (2012).

[24] E. Torrontegui, S. Martínez-Garaot, M. Modugno, X. Chen, and J. G. Muga, Phys. Rev. A 87, 033630 (2013).

[25] S. Martínez-Garaot, E. Torrontegui, X. Chen, M. Modugno, D. Guéry-Odelin, S. Y. Tseng, and J. G. Muga, Phys. Rev. Lett. 111, 213001 (2013).

[26] A. del Campo, Phys. Rev. A 84, 031606(R) (2011).
[27] A. del Campo and M. G. Boshier, Sci. Rep. 2, 648 (2012).

[28] B. Juliá-Díaz, E. Torrontegui, J. Martorell, J. G. Muga, and A. Polls, Phys. Rev. A 86, 063623 (2012).

[29] A. Yuste, B. Juliá-Díaz, E. Torrontegui, J. Martorell, J. G. Muga, and A. Polls, Phys. Rev. A 88, 043647 (2013).

[30] A. del Campo, M. M. Rams, and W. H. Zurek, Phys. Rev. Lett. 109, 115703 (2012).

[31] K. Takahashi, Phys. Rev. E 87, 062117 (2013).

[32] S.-Y. Tseng and X. Chen, Opt. Lett. 37, 5118 (2012).

[33] S. Choi, R. Onofrio, and B. Sundaram, Phys. Rev. A 84, 051601(R) (2011).

[34] S. Choi, R. Onofrio, and B. Sundaram, Phys. Rev. A 86, 043436 (2012).

[35] Y. Li, L. A. Wu, and Z. D. Wang, Phys. Rev. A 83, 043804 (2011).

[36] P. Salamon, K. H. Hoffmann, Y. Rezek, and R. Kosloff, Phys. Chem. Chem. Phys. 11, 1027 (2009).

[37] X. Chen and J. G. Muga, Phys. Rev. A 82, 053403 (2010).

[38] K. H. Hoffmann, P. Salamon, Y. Rezek, and R. Kosloff, Europhys. Lett. 96, 60015 (2011).

[39] A. del Campo, J. Goold, and M. Paternostro, arXiv:1305.3223.

[40] E. Torrontegui, S. Ibáñez, S. Martínez-Garaot, M. Modugno, A. del Campo, D. Guéry-Odelin, A. Ruschhaupt, X. Chen, and J. G. Muga, Adv. At. Mol. Opt. Phys. 62, 117 (2013).

[41] S. Ibáñez, X. Chen, E. Torrontegui, J. G. Muga, and A. Ruschhaupt, Phys. Rev. Lett. 109, 100403 (2012).

[42] K. Takahashi, J. Phys. A: Math. Theor. 46, 315304 (2013).

[43] J. G. Muga, X. Chen, S. Ibañez, I. Lizuain, and A. Ruschhaupt, J. Phys. B 43, 085509 (2010).

[44] H. R. Lewis and W. B. Riesenfeld, J. Math. Phys. 10, 1458 (1969).

[45] R. S. Kaushal and H. J. Korsch, J. Math. Phys. 22, 1904 (1981).

[46] R. S. Kausal and S. C. Misra, J. Math. Phys. 34, 5843 (1993).

[47] R. S. Kaushal and D. Parashar, Phys. Rev. A 55, 2610 (1997).

[48] D. B. Monteoliva, H. J. Korsch, and J. A. Núez, J. Phys. A: Math. Gen. 27, 6897 (1994).

[49] X. Chen, E. Torrontegui, and J. G. Muga, Phys. Rev. A 83, 062116 (2011).

[50] M. Maamache, Phys. Rev. A 52, 936 (1995).

[51] J. G. Muga, J. P. Palao, B. Navarro, and I. L. Egusquiza, Phys. Rep. 395, 357 (2004).

[52] M. A. H. MacCallum, in On Einstein's Path, edited by A. Harvey (Springer, New York, 1999), p. 299.

[53] T. Opatrný and K. Mølmer, New J. Phys. 16, 015025 (2014).

[54] M. P. A. Fisher, P. B. Weichman, G. Grinstein, and D. S. Fisher, Phys. Rev. B 40, 546 (1989).

[55] D. Jaksch, C. Bruder, J. I. Cirac, C. W. Gardiner, and P. Zoller, Phys. Rev. Lett. 81, 3108 (1998).

[56] S. Martínez-Garaot, E. Torrontegui, X. Chen, and J. G. Muga, arXiv:1403.2593. 
[57] F. Boldt, J. D. Nulton, B. Andresen, P. Salamon, and K. H. Hoffmann, Phys. Rev. A 87, 022116 (2013).

[58] D. D'Alessandro, Introduction to Quantum Control and Dynamics (Chapman \& Hall/CRC, Taylor \& Francis Group, London, 2008).

[59] A. Ruschhaupt, Xi Chen, D. Alonso, and J. G. Muga, New J. Phys. 14, 093040 (2012).
[60] X.-J. Lu, X. Chen, A. Ruschhaupt, D. Alonso, S. Guérin, and J. G. Muga, Phys. Rev. A 88, 033406 (2013).

[61] Y. Rezek and R. Kosloff, New J. Phys. 8, 83 (2006).

[62] E. Torrontegui and R. Kosloff, Phys. Rev. E 88, 032103 (2013).

[63] R. Kosloff and T. Feldmann, Phys. Rev. E 82, 011134 (2010).

[64] A. Levy and R. Kosloff, Phys. Rev. Lett. 108, 070604 (2012). 